broad ligament, ${ }^{5-7}$ the uterus,${ }^{8}$ the vagina, ${ }^{9}$ and the testicular and para-testicular tissues. ${ }^{10-12}$ For a diagnosis of Brenner tumour, both integral components (the epithelial and mesenchymal stroma) must be present. ${ }^{278}$

There is only one documented case of extraovarian Brenner tumour of the vagina in the literature. ${ }^{9}$ A similar case, however, has been reported by Buntine $e t a l^{3}$ who called the polyp "benign mixed mullerian tumour of the vagina" and pointed out its resemblance to Brenner tumour. Shevchuk et al, ${ }^{4}$ in their case of "malignant mixed tumour of the vagina", were probably describing a malignant Brenner tumour. The authors commented that the ultrastructural findings of their case confirmed urothelial differentiation and that the tumour appeared to arise from mesonephric remnants. It seems that the reason for the confusion in the nomenclature of this neoplasm is because of its bi- or triphasic nature.

The histogenesis of Brenner tumour is still controversial. ${ }^{2613}$ The important proposed sites of origin include ovarian surface epithelium ${ }^{613}$; remnants of embryonic coelomic epithelium $^{248}$; displaced mesothelium ${ }^{6912}$; rests of Waltbard's cells ${ }^{612}{ }^{13}$; and remnants of mullerian, ${ }^{29-12}$ mesonephric, ${ }^{27101113}$ or wolffian ducts. ${ }^{49101113}$ Other less likely possible sources are as follows: ovarian stroma ${ }^{2813}$; rete ovarii ${ }^{2613}$; rete testis ${ }^{12}$; germ cell (teratomatous) derivation $^{71213}$; granulosa cells ${ }^{1}$; ectopic (accessory) ovarian tissue ${ }^{8}$; ovotestis ${ }^{12}$; and vestibular glands of the vagina. ${ }^{9}$

The presence of the tumour in men and at sites far away from the ovary indicates that this neoplasm is not invariably of ovarian origin. The concept of this neoplasm originating from ovarian coelomic epithelium via a process of wolffian differentiation has been supported by serial reconstruction studies, by its coexistence with other ovarian tumours, and by ultrastructural studies. ${ }^{13}$ Remnants of the wolffian ductal system are known to occur in the broad ligament, cervix and vagina, and must be seriously regarded as an alternative source of the neoplasm in an extraovarian site. ${ }^{2}$ This theory is supported by fact that the tumour is biphasic and that it resembles the transitional epithelium lining the genitourinary tract.

A Brenner tumour of the vagina could therefore be derived directly from wolffian remnants at this site or could originate from mullerian ductal tissue which forms the upper part of the vagina, by a process of wolffian metaplasia.

\footnotetext{
1 Brenner F. Ds oophoroma folliculare. Frank Zeitschr Path 1907;1:150-71.

2 Pschera H, Wikstrom B. Extraovarian tumor coexisting with serous cystadenoma. Gynecol Obstet Invest 1991;31:185-7.

3 Buntine DW, Henderson PR, Biggs JSG. Benign mullerian mixed tumor of the vagina. Gynecol Oncol 1979;8:21-6.

4 Shevchuk MM, Fenoglio CM, Lattes R, Frick HC, Richart RM. Malignant mixed tumor of the vagina probably arising in mesonephric rests. Cancer 1978;42:214-23.

5 Robinson TG. Extraovarian Brenner tumor. Obstet Gynecol 1950;57:890-1.

6 Hampton HL, Huffman HT, Meeks RG. Extraovarian Brenner tumour. Obstet Gynecol 1992;79:844-6.

7 Wagner I, Bettendorf U. Extraovarian Brenner tumour. Case report and review. Arch Gynecol 1980;229:191-6.

8 Arhelger RB, Bocian JJ. Brenner tumor of the uterus. Cancer 1976;38:1741-3.

9 Chen KTK. Brenner tumor of the vagina. Diagn Gynecol Obstet 1981;3:255-8.

10 Goldman RL. A Brenner tumor of the testis. Cancer 1970; 26:853-6.

11 Ross L. Paratesticular Brenner-like tumor. Cancer 1968;21: $722-6$

12 Young RH, Scully RE. Testicular and paratesticular tumors and tumor-like lesions of ovarian common epithelial and mullerian types. A report of four cases and review of the mullerian types. A report of four cases and re

13 Balasa RW, Adcock LL, Prem KA, Dehner LP. The Brenner tumor: a clinicopathological review. Obstet Gynecol 1976; 50:120-8.
}

Department of

Haematology, St Luc

Hospital, Catholic

University of Louvain,

Brussels, Belgium

J L Gala

$\mathrm{J}$ Rodhain

A Ferrant

Department of

Pathology

H Noël

Department of

Haematology, Queen

Astrid Military

Hospital, Brussels,

Belgium

J L Gala

Department of Haematology, Royal

North Shore Hospital, Sydney, Australia

D F F Ma

Correspondence to:

Dr J L Gala, Department of Haematology, Laboratory of Clinical Molecular Biology, Cliniques Universitaires $\mathrm{St}$ Luc, Avenue Hippocrate 10 1200 Brussels, Belgium.

Accepted for publication 26 September 1994

\title{
P-glycoprotein positive, drug resistant invasive lymphoepithelial thymoma: treatment response to chemotherapy with cyclosporin and quinine
}

\author{
J L Gala, H Noël, J Rodhain, D F F Ma, A Ferrant
}

Abstract

A case of invasive drug resistant thymoma, expressing P-glycoprotein, which showed noticeable clinical response to chemotherapy and the multidrug resistance modulating agents cyclosporin and quinine is reported. A 46 year old man presented with severe left shoulder pain and a diagnosis of invasive lymphoepithelial thymoma was made following chest $x$ ray and a computed tomography scan. The patient underwent extensive chemo- therapy without resolution of the tumour. More than $90 \%$ of the malignant epithelial cells were strongly positive for P-glycoprotein and based on this observation, cyclosporin and quinine were added to the chemotherapy regimen. The mediastinal mass completely resolved and the size of the pleural metastasis decreased substantially. The patient, however, died of an intercurrent infection. This case report highlights the feasibility and efficacy of using cyclosporin and quinine in com- 
bination with VAD chemotherapy in the treatment of invasive thymoma.

(f Clin Pathol 1995;48:679-681)

Keywords: Invasive thymoma, multidrug resistance, Pglycoprotein expression.

Thymoma is the commonest type of tumour in the anterior-superior mediastinum, especially in middle-aged or older adults. It is usually a slow growing tumour with benign histology, but it can behave aggressively and invade the surrounding tissues. The standard therapy is local surgery and irradiation. Adjuvant chemotherapy has been used in invasive thymomas. However, recurrences within the thorax are frequent and the mechanisms of resistance to chemotherapy are unclear. We report a case of an invasive drug resistant

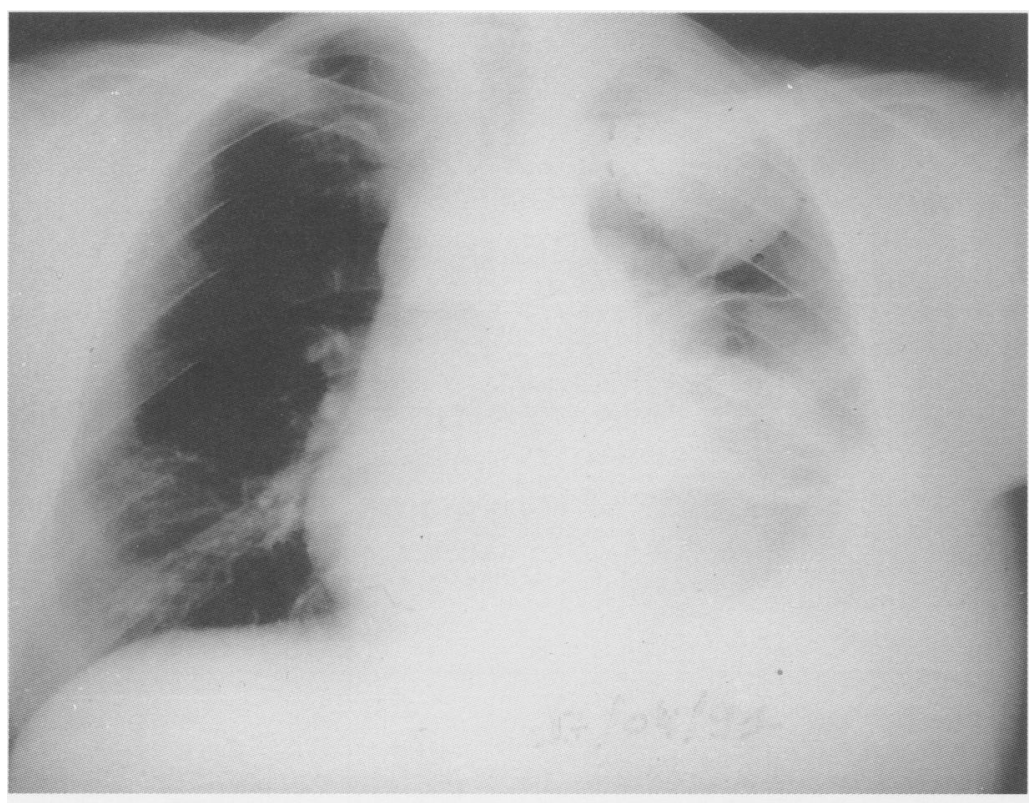

(A)

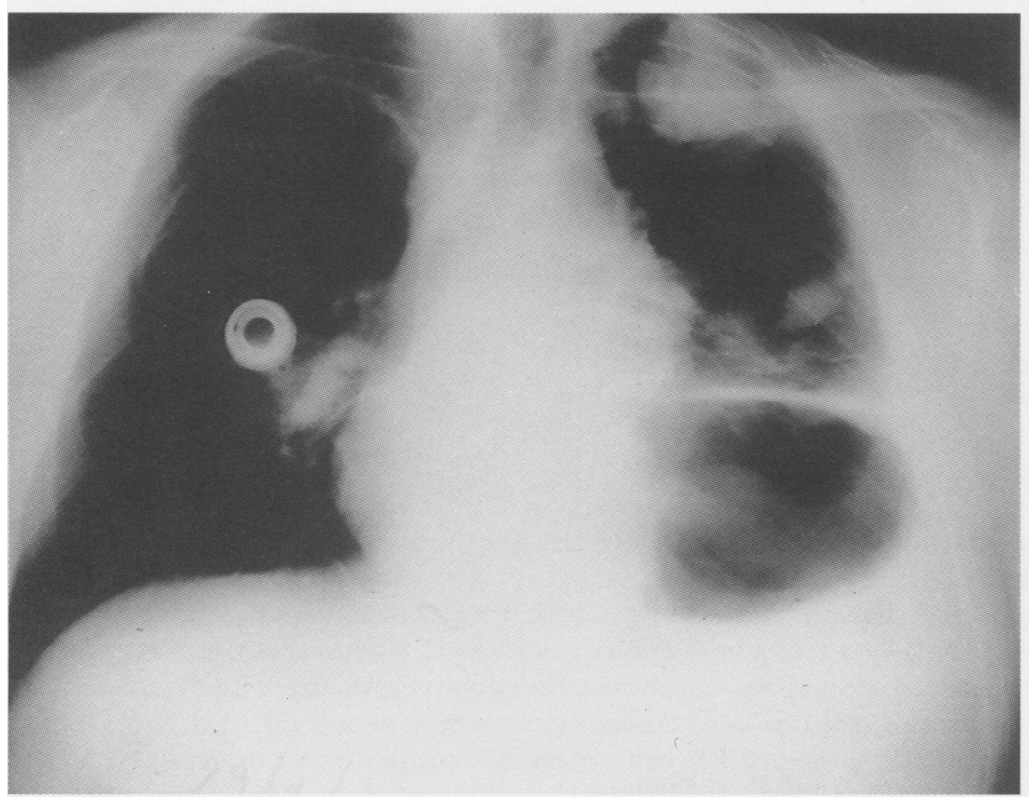

(B)

Figure $1 X$ ray before $(A)$ and after $(B)$ three cycles of VAD chemotherapy and $M D R$ modulating agents (cyclosporin/quinine). thymoma which expressed P-glycoprotein and showed noticeable clinical response to administration of chemotherapy (VAD) and quinine/cyclosporin, multidrug resistance modulating agents.

\section{Case report}

A 46 year old man presented initially with severe left shoulder pain. Chest $x$ ray and a computed tomography scan demonstrated an anterior mediastinal mass invading the pleura and adjacent lung. The diagnosis of invasive lymphoepithelial thymoma was made on a thoracotomy biopsy. Following incomplete resection, the patient was treated with four courses of ProMACE-MOPP (Pro-prednisolone, M-methotrexate, A-doxorubicin, C-cyclophosphamide, E-etoposide, followed by $\mathrm{M}$ mechlorethamine, O-vincristine, $\mathrm{P}$-procarbazine, P-prednisolone). After an initial partial response, rapid tumour progression was seen. Radiotherapy ( $60 \mathrm{~Gy}$ ) was administered to the mediastinum and left hemithorax. This was followed by the administration of etoposide (VP16, $200 \mathrm{mg}$ intravenously on days 1 to 3), cisplatin (200 mg intravenously on day 1 ) and ifosfamide ( $3 \mathrm{~g}$ intravenously on days 1 to 3 ). A total of five courses was given with no response. Severe pancytopenia and two episodes of septicaemia complicated the treatment. Interferon was then administered with the dosage escalated to $10 \times 10^{6}$ units/day five times weekly, in combination with three courses of high dose ifosfamide $\left(1.5 \mathrm{~g} / \mathrm{m}^{2}\right.$ intravenously on days 1 to 5 every four weeks). No decrease in tumour size was observed and interferon was not administered further. At that stage, an isolated pulmonary lesion developed in the upper lobe of the left lung (fig 1A); a fine needle biopsy confirmed the invasion by a lymphoepithelial thymoma. Immunoperoxidase assay on cryostat sections, using the monoclonal antibodies directed against cytokeratin $(39 \mathrm{kDa})$ and $\mathrm{CD} 1$ (Leu 6) identified the thymic malignant epithelial cells and cortical lymphocytes, respectively. More than $90 \%$ of the malignant epithelial cells examined were strongly positive for P-glycoprotein by alkaline phosphataseantialkaline phosphatase assay using the monoclonal antibody JSB-1 as previously reported (fig 2). ${ }^{1}$

Based on these results, the multidrug resistance (MDR) modulating drugs, cyclosporin and quinine, were added to the chemotherapy regimen (VAD, vincristine, doxorubicin and dexamethasone). Cyclosporin was given as an intravenous bolus injection ( $4 \mathrm{mg} / \mathrm{kg}$ ) 24 hours before the VAD infusion on day 0 , then as a continuous infusion until day 6 at a dosage of $8-10 \mathrm{mg} / \mathrm{kg}$. The dose was adjusted to achieve a whole blood cyclosporin concentration above $1500 \mu \mathrm{g} / \mathrm{l}$. Quinine was given as a bolus dose of $7.5 \mathrm{mg} / \mathrm{kg}$ and as a continuous infusion for six days at a dose of $4-7 \mathrm{mg} / \mathrm{kg}$ according to the patient's tolerance. The average daily whole blood cyclosporin concentration and plasma quinine concentration were $1600 \mu \mathrm{g} / \mathrm{l}$ and $8 \mathrm{mg} /$, respectively. After three cycles of therapy, complete resolution of the mediastinal mass 


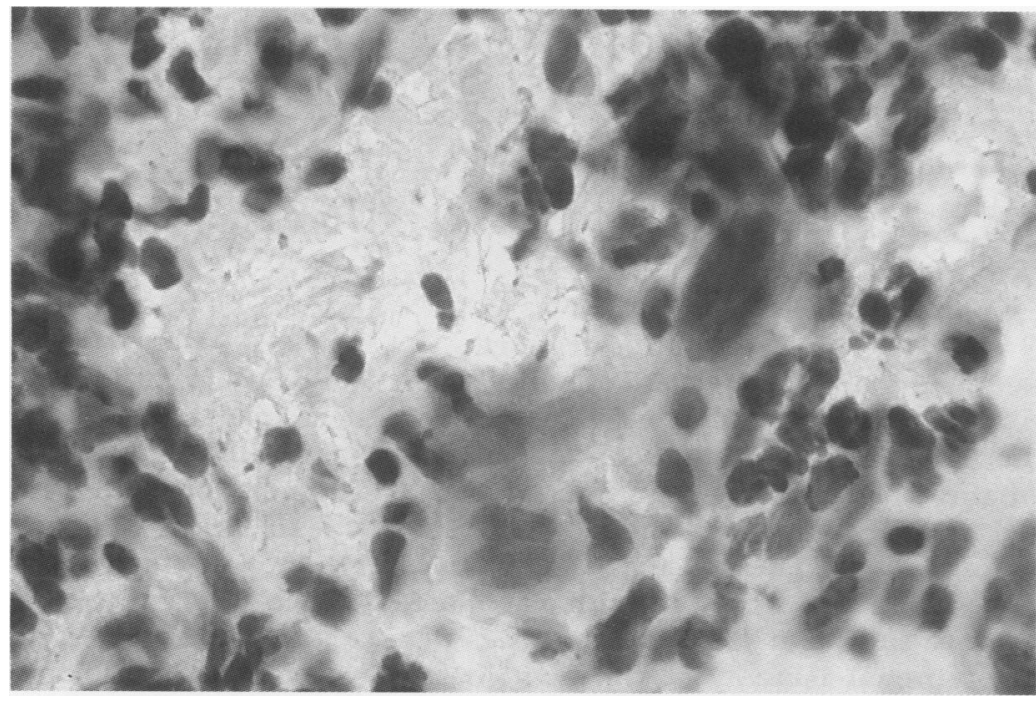

Figure 2 Fine needle biopsy of the thymic lung infiltration: staining of malignant epithelial cells with anti-P-glycoprotein monoclonal antibodies (cryostat section, APAAP using $\mathcal{H S B}-1$; reproduced magnification $\times 365$ ).

and a significant reduction in the size of the pleural metastasis was observed radiologically (figs $1 \mathrm{~A}$ and $1 \mathrm{~B}$ ). The patient received four courses of this therapy without significant side effects, except cinchonism. After completion of the fourth cycle, he died from an intercurrent infection whilst stationed in a neighbouring country. A postmortem examination was not performed.

\section{Discussion}

Although surgery and local radiotherapy are the accepted therapy for thymoma, chemotherapy has been used for invasive and incompletely resected thymoma. Studies have shown that doxorubicin, epirubicin, cyclophosphamide, or ifosfamide, cisplatin, bleomycin, and etoposide are effective chemotherapeutic agents. ${ }^{2-4}$ Overall, the place of chemotherapy and the optimal combination of drugs in the treatment of thymoma are still being evaluated. In lymphoepithelial and epithelial cell predominant thymomas, which are the commonest histological types, ${ }^{5}$ the cortical differentiation of the malignant epithelial cells is correlated with a poor response to therapy and a bad prognosis ${ }^{67}$ but the mechanisms of resistance are unclear.

The MDR, P-glycoprotein phenotype has been shown to be associated with chemotherapy failure in a wide range of haematological malignancies and solid tumours. ${ }^{8}$ The resistance of this cortical thymoma to chemotherapy could be related to P-glycoprotein expression. To our knowledge, this is the first report of P-glycoprotein expression in the malignant epithelial cells of an invasive thymoma. In this patient the accumulated doses of VP16, doxorubicin and vincristine were $4920 \mathrm{mg}, 400 \mathrm{mg}$ and $16 \mathrm{mg}$, respectively. These dose levels are reported to be associated with a high incidence of P-glycoprotein ex- pression following chemotherapy in multiple myeloma. ${ }^{9}$ Because of insufficient initial biopsy material, the issue of whether P-glycoprotein was expressed by the epithelial cells initially or whether this expression was induced by chemotherapy could not be addressed.

Cyclosporin, for multiple myeloma, ${ }^{10}$ and verapamil, for lymphoma, ${ }^{11}$ have been used in the clinical setting as MDR modulating agents. Quinine has been demonstrated to be another non-cytotoxic agent that can act as an MDR modulating agent. ${ }^{12}$ In our patient the tumour was extremely resistant to a wide combination of chemotherapeutic agents, including doxorubicin, vincristine and steroids which are associated in the VAD regimen. This clinical drug resistance together with P-glycoprotein expression by the malignant epithelial cells prompted us to add cyclosporin and quinine to the VAD regimen. Regression of the thymoma was obtained after three cycles of therapy and no significant side effects due to cyclosporin and quinine were observed.

In conclusion, this case report illustrates an invasive thymoma which was resistant to multiple chemotherapeutic agents and expressed high concentrations of P-glycoprotein. It also highlights the feasibility and efficacy of using cyclosporin and quinine in combination with VAD chemotherapy in the treatment of invasive thymoma. Further studies are required to confirm these observations.

This study was supported by the Fonds National de la Recherche Scientifique (Grant Televie no 7.4575.91), the Loterie Nationale and the Salus Sanguinis Foundation.

1 Gala JL, McLachlan J, Michaux JL, Bell DE, Ma DDF. Specificity and sensitivity of immunocytochemistry for detecting P-glycoprotein in haematological malignancies. f Clin Pathol 1994;47:619-24

2 Loehrer PJ Sr, Perez CA, Roth LM, Greco A, Livingston RB, Einhorn LH. Chemotherapy for advanced thymoma. Preliminary results of an intergroup study. Ann Intern Med 1990;113:520-4.

3 Fornasiero A, Daniele O, Ghiotto C, Piazza M, FioreDonati L, Morandi $\mathrm{P}$, et al. Chemotherapy of invasive thymoma. $\mathcal{f}$ Clin Oncol 1990;8:1419-23.

4 Macchiarini P, Chella A, Ducci F, Rossi B, Testi C, Bevilacqua $\mathrm{G}$, et al. Neoadjuvant chemotherapy, surgery, and postoperative radiation therapy for invasive thymoma. postoperative radiation

5 Fukai I, Masaoka A, Hashimoto T, Yamakawa Y, Mizuno $\mathrm{T}$, Tanamura $\mathrm{O}$, et al. An immunohistologic study of the epithelial components of 81 cases of thymoma. Cancer 1992;69:2463-8.

6 Marino M, Muller-Hermelink HK. Thymoma and thymic carcinoma: relation of thymoma epithelial cells to the cortical and medullary differentiation of thymus. Virchows Arch 1985;407:19-49.

7 Pescarmona E, Rendina EA, Venuta F, Ricci C, Ruco LP, Baroni CD. The prognostic implication of thymoma histologic subtyping A study of 80 consecutive cases. $A m$ $f$ Clin Pathol 1990;93:190-5.

8 Arceci RJ. Clinical significance of P-glycoprotein in multidrug resistance malignancies. Blood 1993;81:2215-22.

9 Grogan TM, Spier CM, Salmon SE, Matzner M, Rybski J, Grogan TM, Spier CM, Salmon SE, Matzner M, Rybski J,
Weinstein RS, et al. P-glycoprotein expression in human plasma cell myeloma: correlation with prior chemotherapy. plasma cell myeloma: cor

10 Miller TP, Grogan TM, Dalton WS, Spier CS, Scheper RJ, Salmon SE. P-glycoprotein expression in malignant lymphoma and reversal of clinical drug resistance with chemotherapy plus high-dose verapamil. $\mathcal{f}$ Clin Oncol 1991;9:17-24.

11 Sonneveld P, Durie BG, Lokhorst HM, Marie JP, Solbu G, Suciu S, et al. Modulation of multidrug-resistant myeloma by cyclosporin. Lancet 1992;340:255-9.

12 Gala JL, Zenebergh A, De Bruyere M, Doyen C, Michaux JL. Multidrug resistant multiple myeloma: clinical effects of the association quinine-cyclosporine as a chemosensitizer [abstract]. Blood 1992;80:467a. 